\title{
Rating Prediction based on Social Sentiment from Textual Reviews
}

\author{
R. G. Khedkar \\ Department of computer science and Engineering \\ M.S.Bidve College of Engineering \\ Latur, India
}

\begin{abstract}
In recent years, we've witnessed a flourish of review websites. It presents an excellent chance to share our viewpoints for numerous merchandise we have a tendency to purchase. However, we have a tendency to face the data overloading drawback. A way to mine valuable data from reviews to grasp a user's preferences associated build and correct recommendation is crucial. Ancient recommender systems (RS) take into account some factors, like user's purchase records, product class, and geographic location. During this work, we have a tendency to propose a sentiment-based rating prediction methodology (RPS) to enhance prediction accuracy in recommender systems. Firstly, we have a tendency to propose a social user sentimental measuring approach and calculate every user's sentiment on items/products. Secondly, we have a tendency to not solely take into account a user's own sentimental attributes however additionally take social sentimental influence into thought. Then, we have a tendency to take into account product name, which might be inferred by the sentimental distributions of a user set that replicate customers' comprehensive analysis. At last, we have a tendency to fuse 3 factors-user sentiment similarity, social sentimental influence, and associated item's name similarity into our recommender system to form a correct rating prediction. We have a tendency to conduct a performance analysis of the 3 sentimental factors on a real-world dataset collected from Yelp. Our experimental results show the sentiment will well characterize user preferences that facilitate to enhance the advice performance.
\end{abstract}

\section{Keywords}

Item reputation, Reviews, Rating prediction, Recommender system, Sentiment influence, User sentiment.

\section{INTRODUCTION}

We specialize in the rating prediction task. However, user's rating star-level data isn't perpetually offered on several review websites. Conversely, reviews contain enough careful product data and user opinion data that have nice reference worth for a user's call. Most vital of all, a given user on web site isn't doable to rate each item. Hence, there square measure several unrated things during a user-item-rating matrix. It's inevitable in several rating prediction approaches Review/comment, as we tend to all grasp, is often offered. In such case, it's convenient and necessary to leverage user reviews to assist predicting the unrated things.

Generally, user's interest is stable briefly term, therefore user topics from reviews are often representative. As an example, within the class of Cups \& Mugs, completely different|completely different\} individuals have different tastes. Some individuals concentrate to the standard, some individuals specialize in the worth et al. might valuate comprehensively. Whatever, all of them have their customized topics. Most topic models introduce users'

\author{
S. R. Tandle \\ Department of computer science and Engineering \\ M.S.Bidve College of Engineering \\ Latur, India
}

interests as topic distributions in keeping with reviews contents. They're wide applied in sentiment analysis, travel recommendation, and social networks analysis.

Sentiment analysis is that the most basic and vital add extracting user's interest preferences. In general, sentiment is employed to explain user's own perspective on things. We tend to observe that in several sensible cases, it's additional vital to supply numerical scores instead of binary choices. Generally, reviews square measure divided into 2 teams, positive and negative. However, it's troublesome for purchasers to create a selection once all candidate product replicate positive sentiment or negative sentiment. To create an acquisition call, customers not solely got to grasp whether or not the merchandise is sweet, however conjointly got to savvy smart the merchandise is. It's conjointly in agreement that completely different|completely different $\}$ individuals might have different sentimental expression preferences. as an example, some users value more highly to use "good" to explain associate degree "excellent" product, whereas others might value more highly to use "good" to explain a "just therefore so" product

\section{LITERATURE REVIEW}

\subsection{Circle-based Recommendation in on- line Social Networks \\ Xiwang principle, Harald Steck}

In this paper, online social network data guarantees to extend recommendation accuracy on the far side the capabilities of strictly rating/feedback-driven recommender systems (RS). On higher serve users' activities across totally different domains, several on-line social networks currently support a replacement feature of "Friends Circles" that refines the domain-oblivious "Friends" thought. RS ought to additionally take pleasure in domain-specific "Trust Circles". Intuitively, a user could trust \{different|totally totally different|completely different $\}$ subsets of friends relating to different domains. Sadly, in most existing multi-category rating datasets, a user's social connections from all classes area unit mixed along. This paper presents a shot to develop circle-based RS. We have a tendency to specialize in inferring category-specific social trust circles from offered rating information combined with social network information. We have a tendency to define many variants of weight friends inside circles supported their inferred experience levels. Through experiments on publically offered information, we have a tendency to demonstrate that the planned circle-based recommendation models will higher utilize user's social trust data, leading to inflated recommendation accuracy. 


\subsection{A Matrix resolving Technique with Trust Propagation for Recommendation in Social Networks}

Mohsen Jamali, Martin organic compound

In this paper, recommender systems are getting tools of option to choose the web data relevant to a given user. Cooperative filtering is that the preferred approach to assembling recommender systems and has been with success used in several applications. With the arrival of on-line social networks, the social network primarily based approach to recommendation has emerged. This approach assumes a social network among users and makes recommendations for a user supported the ratings of the users that have direct or indirect social relations with the given user. In concert of their major edges, social network primarily based approaches are shown to scale back the issues with cold begin users. During this paper, we have a tendency to explore a model-based approach for recommendation in social networks, using matrix resolving techniques. Advancing previous work, we have a tendency to incorporate the mechanism of trust propagation into the model. Trust propagation has been shown to be an important development within the social sciences, in social network analysis and in trust-based recommendation. We've conducted experiments on 2 reality information sets, the general public domain Epinions.com dataset and a way larger dataset that we've recently crawled from Flixster.com. Our experiments demonstrate that modeling trust propagation ends up in a considerable increase in recommendation accuracy, above all for cold begin users.

\subsection{Customized Recommendation Combining User Interest and Social Circle He Feng, Guoshuai Zhao}

In this paper, with the arrival and recognition of social network, additional and additional users wish to share their experiences, like ratings, reviews, and blogs. The new factors of social network like social influence and interest supported circles of friends bring opportunities and challenges for recommender system (RS) to resolve the cold begin and scantiness drawback of datasets. A number of the social factors are employed in RS, however haven't been totally thought of. During this paper, 3 social factors, personal interest, social interest similarity, and social influence, fuse into a unified customized recommendation model supported probabilistic matrix resolving. The issue of non-public interest will build the RS advocate things to fulfill users' individualities, particularly for knowledgeable users. Moreover, for cold begin users, the social interest similarity and social influence will enhance the intrinsic link among options within the latent house. We have a tendency to conduct a series of experiments on 3 rating datasets: Yelp, MovieLens, and Douban film. Experimental results show the planned approach outperforms the prevailing RS approaches.

\subsection{Recommendation via User's temperament and Social discourse}

He Feng and Xueming Qian

In this paper, with the arrival and recognition of social network, additional and additional users wish to share their experiences, like ratings, reviews, and blogs. The new factors of social network like social influence and interest supported circles of friends bring opportunities and challenges for recommender system (RS) to resolve the cold begin and scantiness drawback of datasets. a number of the social factors are employed in RS, however haven't been totally thought of. During this paper, 3 social factors, personal interest, social interest similarity and social influence, fuse into a unified customized recommendation model supported probabilistic matrix resolving. The issue of non-public interest will build the RS advocate things to fulfill users' individualities, particularly for knowledgeable users. Moreover, for cold begin users, the social interest similarity and social influence will enhance the intrinsic link among options within the latent house. We have a tendency to conduct a series of experiments on real rating datasets. Experimental results show the planned approach outperforms the prevailing RS approaches.

\subsection{Nonlinear latent resolving by Embedding Multiple User Interests \\ Jason Edward Weston, Ron J. Weiss}

In this paper, classical matrix resolving approaches to cooperative filtering learn a latent vector for every user and every item, and suggestions area unit scored via the similarity between 2 such vectors, that area unit of an equivalent dimension. During this work, we have a tendency to area unit motivated by the intuition that a user may be a rather more sophisticated entity than any single item, and can't be delineate by an equivalent illustration. Hence, the variability of a user's interests might be higher captured by a additional complicated illustration. We have a tendency to propose to model the user with a richer set of functions, specifically via a collection of latent vectors, wherever every vector captures one in every of the user's latent interests or tastes. the general recommendation model is that then nonlinear wherever the matching score between a user and a given item is the most matching score over every of the user's latent interests with relevance the item's latent illustration. We have a tendency to describe an easy, general and economical algorithmic rule for learning such a model, and apply it to massive scale, real world datasets from YouTube and Google Music, wherever our approach outperforms existing techniques.

\subsection{Personalized Recommendation Combining User Interest and Social Circle} Xueming Qian, Guoshuai Zhao

In this paper, with the advent and popularity of social network, more and more users like to share their experiences, such as ratings, reviews, and blogs. The new factors of social network like interpersonal influence and interest based on circles of friends bring opportunities and challenges for recommender system (RS) to solve the cold start and sparsity problem of datasets. Some of the social factors have been used in RS, but have not been fully considered. In this paper, three social factors, personal interest, interpersonal interest similarity, and interpersonal influence, fuse into a unified personalized recommendation model based on probabilistic matrix factorization. The factor of personal interest can make the RS recommend items to meet users' individualities, especially for experienced users. Moreover, for cold start users, the interpersonal interest similarity and interpersonal influence can enhance the intrinsic link among features in the latent space. We conduct a series of experiments on three rating datasets: Yelp, MovieLens, and Douban Movie. Experimental results show the proposed approach outperforms the existing RS approaches. 


\subsection{Personalized Recommendation Based on Reviews and Ratings Alleviating the Sparsity Problem of Collaborative Filtering Jingnan Xu, Xiaolin Zheng}

In this paper, with the development of e-commerce, shopping on-line is becoming more and more popular. When we need to decide whether to purchase a product or not on line, the opinions of others become important. The convenience of new web technologies enables us to freely express our opinions and reviews for various products we have purchased which leads to a serious problem, information overloading. How to mine these review data to understand customers' preferences and make recommendations is crucial to merchants and researchers. Traditional collaborative filtering $(\mathrm{CF})$ algorithm is one of the most successful recommendation system technologies. The core idea of CF algorithm is to recommend products based on other people who have similar tastes with target users. However, the ability of CF is limited by the sparsity problem, which is very common in reality. The reason derives from the fact that traditional CF method only takes users' ratings into account. In this paper, we propose a new personalized recommendation model, i.e. topic model based collaborative filtering (TMCF) utilizing users' reviews and ratings. We exploit extended LDA model to generate topic allocations for each review and then obtain each user's preference. Moreover, a new metric is designed to measure similarity between users alleviating the sparsity problem to a large extent. Finally, recommendations are made based on similar users' ratings. Experiments on seven data sets indicate better prediction accuracy than other traditional and state-ofthe-art methods with substantial improvement in alleviating the sparsity problem.

\subsection{Personalized Recommendation Based on Reviews and Ratings Alleviating the Sparsity Problem of Collaborative Filtering Jingnan Xu, Xiaolin Zheng}

In this paper, with the development of e-commerce, shopping on-line is becoming more and more popular. When we need to decide whether to purchase a product or not on line, the opinions of others become important. The convenience of new web technologies enables us to freely express our opinions and reviews for various products we have purchased which leads to a serious problem, information overloading. How to mine these review data to understand customers' preferences and make recommendations is crucial to merchants and researchers. Traditional collaborative filtering $(\mathrm{CF})$ algorithm is one of the most successful recommendation system technologies. The core idea of CF algorithm is to recommend products based on other people who have similar tastes with target users. However, the ability of CF is limited by the sparsity problem, which is very common in reality. The reason derives from the fact that traditional CF method only takes users' ratings into account. In this paper, we propose a new personalized recommendation model, i.e. topic model based collaborative filtering (TMCF) utilizing users' reviews and ratings. We exploit extended LDA model to generate

\section{EXISTING SYSTEM}

In our daily life, customers are possibly to shop for those product with highly-praised reviews. That is, customers are additional involved concerning item's name that reflects consumers' comprehensive analysis supported the intrinsic price of a particular product. To get the name of a product, sentiment in reviews is critical. Normally, if item's reviews mirror positive sentiment, the item could also be with sensible name to an excellent extent. Oppositely, if item's reviews are packed with negative sentiment, then the item is to be with dangerous name. To a given product, if we all know user sentiment, we will infer the name and even the great ratings. After we search information superhighway for getting, each positive reviews and negative reviews are valuable to be as reference. For positive reviews, we will understand the benefits of a product. For negative reviews, we will get the shortcomings just in case of being cheated. Thus it's price to explore those reviewers World Health Organization have obvious and objective perspective on things. We have a tendency to observe that reviewers' sentiment can influence others: if a reviewer has clear like and dislike sentiment, alternative users can pay abundant attention to him/her. However, user's sentiment is difficult to predict and also the unpredictability of social sentimental influence makes an excellent problem in exploring social users.

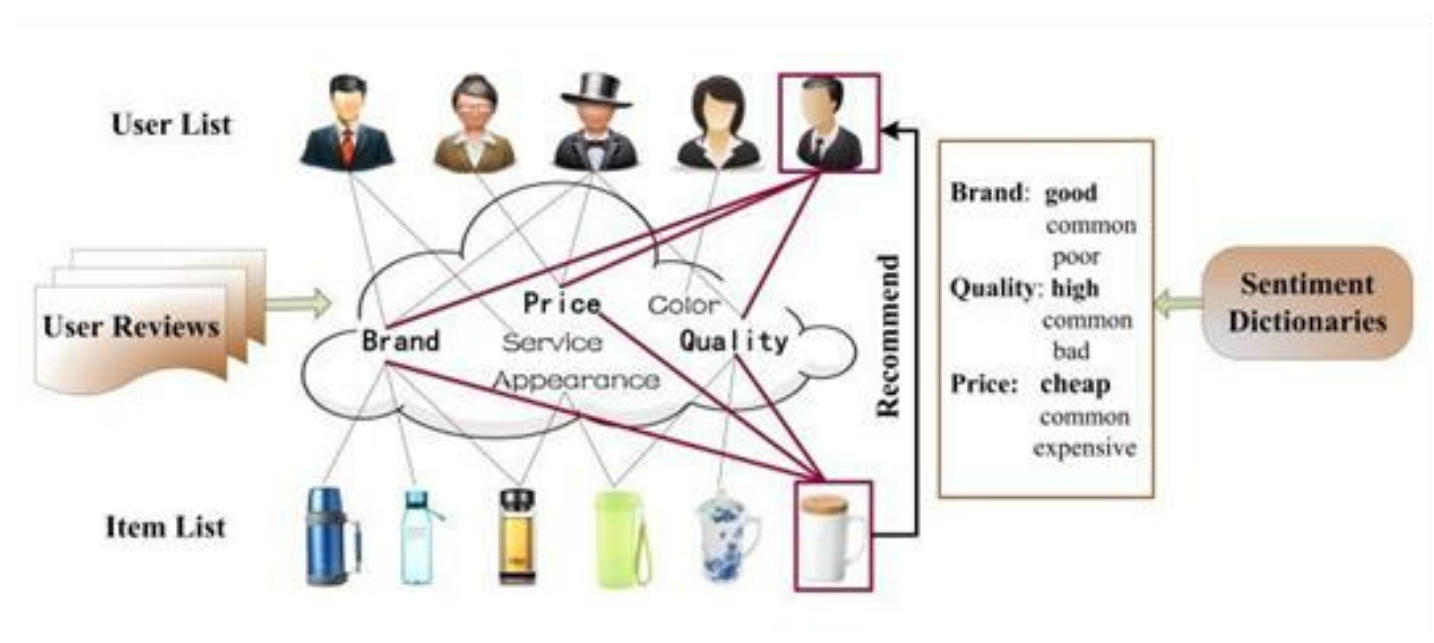

Fig 1: System Architecture 


\section{PROPOSED SYSTEM}

We propose a sentiment-based rating prediction technique within the framework of matrix resolving. In our work, we have a tendency to create use of social users' sentiment to infer ratings. First, we have a tendency to extract product options from user reviews. Then, we discover out the sentiment words that are wont to describe the merchandise options. Besides, we have a tendency to leverage sentiment dictionaries to calculate sentiment of a particular user on associate degree item/product. The main contributions of our approach are as follows: 1) we have a tendency to propose a user sentimental measure approach that is predicated on the mined sentiment words and sentiment degree words from user reviews. Besides, some climbable applications are planned. As an example, we have a tendency to explore however the mined sentiment unfold among users' friends. What's additional, we have a tendency to leverage social users' sentiment to infer item's name that showed nice improvement in accuracy of rating prediction. 2) We have a tendency to create use of sentiment for rating prediction. User sentiment similarity focuses on the user interest

\section{CONCLUSION AND FUTURE WORK}

In this system, a recommendation model is projected by mining sentiment info from social users' reviews. We have a tendency to fuse user sentiment similarity, social sentiment influence, and item name similarity into a unified matrix factorization framework to attain the rating prediction task. Above all, we have a tendency to use social users' sentiment to denote user preferences. Besides, we have a tendency to build a brand new relationship named social sentiment influence between the user and friends that reflects however users' friends influence users during a sentimental angle. What's a lot of, as long as we have a tendency to get user's matter reviews, we are able to quantitively live user's sentiment, and that we leverage items' sentiment distribution among users to infer item's name. The experiment results demonstrate that the 3 sentimental factors create nice contributions to the rating prediction. Also, it shows vital enhancements over existing approaches on a real-world dataset. In our future work, we are able to think about a lot of linguistic rules once analyzing the context, and that we will enrich the sentiment dictionaries to use fine-grained sentiment analysis. Besides, we are able to adapt or develop alternative hybrid factorization models like tensor factorization or deep learning technique to integrate phrase-level sentiment analysis.

\section{REFERENCES}

[1] R. Salakhutdinov, and A. Mnih, "Probabilistic matrix factorization," in NIPS, 2008.

[2] X. Yang, H. Steck, and Y. Liu, "Circle-based recommendation in online social networks, " in Proc. 18th ACM SIGKDD Int. Conf. KDD, New York, NY, USA, Aug. 2012, pp. 1267-1275.

[3] M. Jiang, P. Cui, R. Liu, Q. Yang, F. Wang, W. Zhu, and S. Yang, "Social contextual recommendation," in proc. 21st ACM Int. CIKM, 2012, pp. 45-54.

[4] M. Jamali and M. Ester, "A matrix factorization technique with trust propagation for recommendation in social networks," in Proc. ACM conf. RecSys, Barcelona, Spain. 2010, pp. 135-142.

[5] Z. Fu, X. Sun, Q. Liu, et al., "Achieving Efficient Cloud Search Services: Multi-Keyword Ranked Search over Encrypted Cloud Data Supporting Parallel Computing," IEICE Transactions on Communications, 2015 , 98(1):190-200.

[6] G. Ganu, N. Elhadad, A Marian, "Beyond the stars: Improving rating predictions using Review text content," in 12th International Workshop on the Web and Databases (WebDB 2009). pp. 1-6.

[7] J. Xu, X. Zheng, W. Ding, "Personalized recommendation based on reviews and ratings alleviating the sparsity problem of collaborative filtering," IEEE International Conference on e-business Engineering. 2012, pp. 9-16.

[8] X. Qian, H. Feng, G. Zhao, and T. Mei, "Personalized recommendation combining user interest and social circle," IEEE Trans. Knowledge and data engineering. 2014, pp. 1763-1777 